\title{
VAC/VI Regimen
}

National Cancer Institute

\section{Source}

National Cancer Institute. VACIVI Regimen. NCI Thesaurus. Code C148284.

A reg imen consisting of vincristine, dactinomycin and cyclophosphamide, alternating with vincristine and irinotecan that can be used for the treatment of rhabdomyosarcoma. 